\title{
Hybrid
}

Revue des arts et médiations humaines

\section{Habiter les écologies fantasy : la littérature face au dispositif vidéoludique}

\section{Pierre-Louis Patoine}

\section{(2) OpenEdition}

1 Journals

\section{Édition électronique}

URL : https://journals.openedition.org/hybrid/309

DOI : 10.4000/hybrid.309

ISSN : 2276-3538

Traduction(s) :

Inhabiting fantasy ecologies: literature meets video games - URL : https://journals.openedition.org/ hybrid/398 [en]

Éditeur

Presses universitaires de Vincennes

Référence électronique

Pierre-Louis Patoine, « Habiter les écologies fantasy : la littérature face au dispositif vidéoludique », Hybrid [En ligne], 5 | 2018, mis en ligne le 18 décembre 2018, consulté le 14 avril 2022. URL : http:// journals.openedition.org/hybrid/309; DOI : https://doi.org/10.4000/hybrid.309

Ce document a été généré automatiquement le 14 avril 2022.

Revue Hybrid 


\title{
Habiter les écologies fantasy : la littérature face au dispositif vidéoludique
}

\author{
Pierre-Louis Patoine
}

$1 \mathrm{Au} \mathrm{XIX}^{\mathrm{e}}$ siècle, les innovations dans les domaines de l'optique, de la chimie et de la mécanique convergent pour permettre l'émergence de la photographie. Cette technique se révélera porteuse d'un art qui se prête d'emblée au récit: le cinéma. Art natif au médium photographique, celui-ci se dégagera peu à peu des codes hérités des formes narratives alors dominantes, codes théatraux et littéraires, pour établir sa propre esthétique, ses propres conventions, qui auront en retour un impact majeur sur la littérature et le théâtre tout au long du $\mathrm{xx}^{\mathrm{e}}$ siècle.

2 Les médias numériques suivent un parcours comparable. De l'algorithmique babylonienne, euclidienne, perse et arabe aux métiers à tisser Jacquard avec leurs cartes perforées (1801), de la machine analytique de Charles Babbage imaginée en 1834 à la machine de Turing conçue un siècle plus tard, de la mécanographie de la Belle Époque à l'invention du transistor puis du circuit intégré, l'histoire de l'informatique aura combiné de multiples branches des mathématiques, de la logique et de la physique pour permettre l'avènement des médias numériques dans les dernières décennies du $\mathrm{xx}^{\mathrm{e}}$ siècle. Comme la photographie aura donné naissance au cinéma (et pas seulement au roman-photo ou au théâtre filmé), l'informatique donnera naissance au jeu vidéo dans les années 1960 et 1970 (et pas seulement à la littérature électronique ou au film interactif). Celui-ci s'intéressera rapidement et de manière décisive au récit, développant des manières de raconter des histoires, des stratégies narratives et stylistiques qui se cristallisent dans les années 1980 et 1990, alors que ce média connaît une véritable poussée de croissance. Cette croissance s'étant maintenue au cours des premières décennies du Xxi ${ }^{e}$ siècle, il semble aujourd'hui essentiel de comprendre quels impacts cette forme d'art, native du domaine informatique, a sur notre culture, et notamment sur la manière dont nous pratiquons la littérature. Que devient cette 
dernière au sein d'un environnement technique toujours plus imprégné d'informatique?

3 Nous proposons ici une réponse partielle et ponctuelle à cette question, à partir de l'étude d'un champ d'influence précis, que circonscrit une remarque de N. Katherine Hayles. Dans Electronic Literature, elle écrit en effet, à propos des formes narratives électroniques et imprimées, «qu'elles se concurrencent et coopèrent non seulement en termes de design, de style, et de contenu thématique, mais également en termes de préférences du lecteur et des modes de son implication ${ }^{1}$ ». Ce sont ces préférences et ces modes d'implication du joueur et du lecteur qui nous intéresseront ici. Il ne s'agira donc pas tant d'évaluer la manière dont informatique et littérature échangent thèmes et images, que de comprendre comment leur rencontre favorise des usages particuliers du récit et de la fiction.

4 C'est sur le terrain de la fantasy, un genre partagé entre le littéraire et le vidéoludique, que nous étudierons ces usages et ces modes d'implication. La fantasy constitue une matrice d'influence qui s'organise autour d'un principe à la fois esthétique, narratif, thématique, ludique et lectorial, celui de la création et de l'exploration d'univers secondaires, imaginaires ou virtuels. Ces univers ouvrent vers une pratique immersive du texte qui, issue de la littérature et devenue centrale dans le jeu vidéo, participe en retour à la construction de l'horizon d'attente des lecteurs au xxi ${ }^{\mathrm{e}}$ siècle et à la valorisation rétrospective des textes fondateurs de la culture geek et vidéoludique.

Cette matrice d'influence prend forme avec la constitution progressive de la fantasy moderne dans les décennies 1930-1970, au fil des œuvres d'auteurs comme Robert E. Howard, Fritz Leiber, J. R. R. Tolkien, Michael Moorcock ou Ursula Le Guin. Celles-ci formeront le cadre esthétique et imaginaire dans lequel Gary Gygax et Dave Arneson planteront leur jeu de rôle Donjons et Dragons, qui paraît en 1974. À cette époque, la première grande génération de programmeurs traverse l'adolescence ou les débuts de l'âge adulte. Elle se réunit en groupes qui jouent à Donjons et Dragons, se plongent dans les œuvres que nous venons de mentionner, et trouvent dans l'informatique un outil puissant pour prolonger l'«expérience fantas $y^{2} »$ : c'est le début de la fiction interactive, des text adventures tels que Adventure (1975) et Zork (1977), qui posent les premiers jalons de ce qui deviendra petit à petit le jeu vidéo de rôle. Avec les années 1980 , cette matrice fantasy se constitue en champ intermédial sous l'impulsion d'une communauté de passionnés créatifs et entreprenants. Avec Warlock of Firetop Mountain (1982), Steve Jackson et Ian Livingston croisent jeu de rôle, programmation et roman jeunesse pour créer le premier « livre dont vous êtes le héros ». Deux ans plus tard, l'éditeur TSR lance la série de romans Dragonlance (coécrits par Margaret Weis et Tracy Hickman) pour accompagner ses modules de jeu Donjons et Dragons. En 1988, et toujours chez TSR, c'est au tour du jeu vidéo Pool of Radiance et de l'univers partagé des «Forgotten Realms» d'inspirer un roman (du même titre, paru en 1989). Qu'ils soient littéraires ou vidéoludiques, ces récits fantasy ont quelque chose en commun : ils sont construits autours d'univers explorables, souvent partagés, et favorisent une forme d'immersion spatiale, géographique ou environnementale.

Bien entendu, l'exploration et l'immersion spatiale ne sont pas des formes d'implication que la fantasy moderne invente. Il y a en Occident une longue tradition de " récits spatiaux », qu'on pourrait faire remonter à L'Épopée de Gilgamesh, à L'Illiade et à L'Odyssée, à Beowulf, aux formes diverses du récit de voyage, à la pastorale, à l'utopie, puis au roman d'aventure. Mais aux xxe et xxI siècles, c'est sans doute la fantasy (et la 
science-fiction), qui mobilisent avec le plus de force les rapports d'immersion, d'exploration et d'habitation que nous tenterons de décrire ici.

\section{Le jeu vidéo de rôle}

7 S'il peut être considéré, avec Marie-Laure Ryan, comme «l'un des domaines les plus productifs d'activité narrative au sein des médias numériques ${ }^{3}$ ", le jeu vidéo inclut une variété de genres qui sont loin d'être également intéressés par le récit. Parmi ces genres, le jeu vidéo de rôle, héritier direct du jeu de rôle de table Donjons et Dragons et communément appelé RPG (pour Role Playing Game, nous conserverons cet acronyme), s'est montré particulièrement apte à proposer des histoires complexes, le plus souvent structurées autour d'une quête qui fait évoluer un héros ou un groupe de héros. Comme l'écrit James Newman, «le récit, la richesse et la complexité des dialogues et des personnages dans les jeux vidéo de rôle sont des éléments vitaux de ce genre des plus littéraires", un genre qui "créé le lien entre les jeux vidéo et d'autres formes littéraires $»^{4}$.

8 C'est donc essentiellement au RPG que nous nous intéresserons, et aux façons dont celui-ci répond au désir archaïque d'échapper temporairement aux contingences de la vie quotidienne pour habiter un "monde meilleur", pour s'immerger dans un environnement merveilleux ${ }^{5}$. Ce n'est pas un hasard si, comme le remarquent King et Borland, les trois paramètres qui se croisent dans les années 1970 pour rendre possible cette échappée (littérature fantasy, jeu de rôle, informatique), nous parlent, chacun à sa manière, de magie ; d'une forme de transcendance, par l'imaginaire et la technologie, du quotidien et de la condition humaine ${ }^{6}$.

\section{La technique, production de monde}

9 C'est donc dans les années 1970 et au sein de la matrice d'influence fantasy que les techniques informatiques (programmation, interactivité) se combinent aux techniques ludiques (règles et systèmes de jeu) et littéraires (conventions génériques, formes narratives et figuratives) pour simuler des univers secondaires explorables qui élargissent au quotidien notre umwelt et participent ainsi au devenir biologique et esthétique de l'humain et de son habitat. Comme l'écrit Montani :

[...] la forme de vie humaine est technique dès son origine, en ceci qu'elle s'extériorise de façon constitutive dans un prolongement non organique qui influe de façon plus ou moins profonde - mais en tous cas décisive - sur l'organisation de sa sensibilité et, coessentiellement, sur le monde des choses que rencontre et qu'élabore cette sensibilité?.

Les mondes fantasy apparaissent en effet comme des extensions non organiques de notre environnement, des mondes qui modulent nos sensibilités en nous fournissant notamment refuges et abris. Comme une maison permet d'extérioriser dans une forme technique la fonction de régulation de notre température interne (entre autres), ces mondes fictionnels permettent de déléguer à des artefacts iconiques et symboliques, socialement partagés, des fonctions biologiques initialement attachées au «corps animal », notamment des fonctions homéostatiques de régulation du stress et de la tension. La création d'habitats fictionnels prolonge ainsi le processus d'hominisation que décrit Leroi-Gourhan dans Le Geste et la Parole ${ }^{8}$. 
11 C'est notamment par sa dimension spatiale que le récit littéraire permet des formes d'habitation fictionnelle. Arguant que les pratiques du récit exploitent des structures cérébrales et cognitives développées lors de l'évolution de l'espèce humaine, Christopher Collins écrit : "Il est possible d'établir une analogie entre écouter un récit et se déplacer d'objet en objet au sein d'un espace, simplement, on n'évolue plus au sein d'un paysage, mais d'un environnement sonore et, au lieu d'un flux optique, nous traitons un flux acoustique 9 . " Le récit nous guide ainsi à travers des environnements plus ou moins hospitaliers, grâce à des catégories linguistiques qui traduisent des aspects spécifiques de cette navigation spatiale. Le nom - qui est central, nous le verrons, à la création d'univers secondaires et au spectacle environnemental équivaudrait ainsi, selon Collins, à l'objet de la perception focale, aux figures que notre regard détache sur l'arrière-plan périphérique, lorsque nous explorons nos alentours ${ }^{10}$. L'expérience environnementale du récit verbal (et, a fortiori, du récit vidéoludique) rejoue l'oscillation entre sécurité $d u$ refuge et stress de l'exploration ${ }^{11}$. Nous reviendrons sur ce point, mais notons dès à présent que cette oscillation peut correspondre de manière complexe au binôme narration-description. Pour le géographe Yi-Fu Tuan, dans la société primitive, le récit serait lié à la pratique de la chasse et de l'exploration (qui suivent le schéma narratif classique de la quête épique), alors que la cueillette, qui se fait dans les environs de l'abri, rappelle plutôt la description minutieuse ${ }^{12}$.

12 Cette description-refuge, dans la fantasy, apparaît souvent comme un moment d'apaisement, libéré des exigences de l'action, de l'enchaînement syntaxique des causes et des effets, bref de l'avancée et de la résolution du récit. Elle présente des paysages pittoresques, des environnements spectaculaires que le lecteur aime habiter temporairement. Le développement des sciences informatiques a permis tout à coup de rendre visibles, explorables, habitables ces mondes merveilleux, jusque-là cantonnés aux jeux imaginaires de la littérature. Il nous a fourni une manière d'interagir avec ces mondes, sans les contraintes (ni les avantages) du jeu de rôle de table. Aussitôt ouvert, le champ informatique met donc son caractère futuriste au service de fantasmes ancestraux de transcendance, de rêves mythiques, épiques ou héroïques. Shamans électroniques, les premiers programmeurs nous auront permis d'entrer dans des mondes féériques riches en sensations, nous invitant à traverser la surface hypnotique de l'écran pour explorer des univers enracinés dans une tradition littéraire dont Tolkien est sans doute le plus célèbre contributeur $\mathrm{au} \mathrm{Xx}^{\mathrm{e}}$ siècle.

\section{Tolkien, la fantasy et les mondes secondaires}

13 Au sein de la matrice d'influence dont nous cherchons à identifier les lignes de force, Tolkien n'est pas aussi central que sa visibilité « grand public » peut le laisser supposer. Mais il se prête commodément au discours universitaire : professeur d'ancien anglais à Oxford, il nous a laissé des réflexions sur sa pratique, et son œuvre convient aisément à l'analyse littéraire telle que nous la pratiquons, alors que, par exemple, l'écriture pulp de Robert E. Howard, le père de Conan, déroute au contraire notre regard et nos outils critiques. Jeune Texan écrivant pour gagner sa vie, Howard incarne parfaitement «l'autre branche» de la fantasy, énergique et irrévérencieuse, qui jouera un rôle majeur dans l'élaboration de l'imaginaire de Donjons et Dragons, puis du jeu vidéo. Howard sera d'ailleurs le premier auteur de fantasy moderne à publier une « histoire » 
de son univers fictionnel, "The Hyborian Age ${ }^{13}$ ». Malgré ce geste fort, le monde d'Hyperborea parait subordonné à son héros Conan : il est avant tout une scène pour ses aventures.

À l'inverse, chez Tolkien, la création de l'univers secondaire prime, et son œuvre apparaît essentiellement comme une manière de l'habiter, d'y passer du temps. Bien que nous les ayons d'abord visités à travers un roman pour enfant (The Hobbit, 1937), puis dans une « suite pour adulte » (The Lord of the Rings, 1954-1955), la Terre du Milieu et l'univers d'Ea naissent dans les tranchées de la Première Guerre mondiale, alors que Tolkien se réfugie dans l'imaginaire et prend les premières notes, écrit les premiers poèmes de ce qui deviendront les douze volumes de L'Histoire de la Terre du Milieu et le Silmarillion. Ce dernier raconte l'histoire d'Ea, de sa création jusqu'au début de son troisième âge (au cours duquel se déroulent Le Hobbit et Le Seigneur des anneaux). Cette histoire émerge par fragments : le Silmarillion est un assemblage de courtes histoires, héroïques, mythiques, peu psychologiques mais très généalogiques, très géographiques, territoriales et environnementales. Il est essentiel pour suivre et comprendre le Silmarillion de se référer fréquemment à la carte placée à l'entrée du texte.

L'œuvre de Tolkien est donc, dès ses débuts, ontologiquement orientée vers les objets, les territoires, l'environnement. Cette orientation est liée à des options génériques ; on le voit lorsque Tolkien explique pourquoi le genre dramatique se prête mal, selon lui, à la fantasy: "You are, for instance, likely to prefer characters, even the basest and dullest, to things. Very little about trees as trees can be got into a play ${ }^{14}$.» Quoique discutable, notamment dans le contexte du théâtre contemporain, cette citation montre surtout que le projet romanesque de Tolkien est justement de faire une place au non-humain, au non-psychologique, à l'environnemental. Nous verrons que le jeu vidéo de rôle, parce qu'il accorde une place privilégiée au spectacle environnemental, partage cette orientation. Le récit fantasy selon Tolkien combine donc ontologie, environnement et immersion :

Fairy-stories are not in normal English usage stories about fairies or elves, but stories about Fairy, that is Faërie, the realm or state in which fairies have their being. Faërie contains many things besides elves and fays, and besides dwarfs, witches, trolls, giants, or dragons : it holds the seas, the sun, the moon, the sky ; and the earth, and all things that are in it: tree and bird, water and stone, wine and bread, and ourselves, mortal men, when we are enchanted ${ }^{15}$.

La fantasy est ici clairement définie à travers sa densité écologique, sa capacité à créer des univers secondaires complets et habitables. Les contes de fées ne sont pas des histoires à propos de créatures particulières, mais de leur monde, de leur plan d'existence défini en termes d'éléments écologiques (mers, soleil, lune, ciel, terre, arbres, oiseaux...) et d'artefacts fondamentaux de la culture humaine, pain et vin ; car les humains sont aussi invités à parcourir ce monde, à une condition importante : qu'ils soient enchantés. Cet enchantement ne correspond-il pas à l'immersion environnementale qui caractérise la fantasy littéraire et vidéoludique?

\section{Aspect spatial du jeu vidéo}

En donnant naissance au jeu vidéo, l'informatique aura permis de renforcer ce lien qu'établit la littérature fantasy entre monde secondaire et immersion ou enchantement. En effet, pour le ludologue Espen Aarseth, les jeux vidéo, médias 
immersifs et hypnotiques, "se consacrent essentiellement à la représentation et à la négociation de l'espace ${ }^{16}$ ", alors que pour Henry Jenkins, "les designers de jeux ne racontent pas simplement des histoires; ils conçoivent des mondes et sculptent l'espace ${ }^{17} »$. Bien qu'il serait erroné de réduire le domaine vidéoludique à sa dimension spatiale, et que des jeux de gestion d'équipes sportives tels que Football Manager (2004-2018) ou de simulation amoureuse comme la série des Tokimeki Memorial (1994-2006) montrent que cette dimension ne constitue pas un impératif catégorique du médium ${ }^{18}$, les affirmations de Aarseth et de Jenkins sont particulièrement pertinentes dans le contexte des jeux vidéo de rôle qui tendent vers le fantasy. En effet, «le spectacle environnemental [...] est particulièrement important dans les jeux se déroulant dans les espaces appartenant à la high fantasy, qui créent souvent des mondes étendus composés de paysages aux styles variés ${ }^{19}$ ».

L'exploration plus ou moins orientée de ces paysages organise le déploiement du récit de la plupart des RPG et des fictions interactives. Cette centralité de l'exploration est évidente dès la première fiction interactive, Adventure, créée en 1975 par le programmeur Will Crowther, un joueur de Dungeons et Dragons, mais peut-être surtout un spéléologue averti qui a voulu recréer le plaisir de l'exploration pour ses deux filles. Même si la première version de ce jeu, nommée Colossal Cave, est entièrement textuelle, sans interface graphique, sa nature reste spatiale et exploratoire, le monde décrit étant inspiré par des endroits spécifiques, souvent spectaculaires, des Mammoth Caves du Kentucky $^{20}$. Mais la simulation de cavernes à explorer ne semble pas suffisante à Crowther pour retenir l'attention de ses filles. Il place donc des éléments qui motiveront et orienteront l'exploration de cet espace virtuel : cinq trésors à découvrir, un serpent qui fait obstacle à la progression du joueur, le fameux labyrinthe formé des "twisty little passages, all alike» et ainsi de suite. Ces objectifs et ces obstacles, qui forment l'aspect narratif, orienté et téléologique du jeu, semblent relativement indépendants du plaisir de l'exploration, comme le souligne Nick Montfort, qui écrit à propos de ce jeu : « Il y a aussi la sensation d'explorer un monde ou un espace nouveau, indépendant des événements se déroulant dans cet espace et qui sont narrés. Le plaisir de cette exploration n'est pas lié aux énigmes et à leur résolution ${ }^{21}$. " Il y a donc une tension productive entre, d'une part, le développement du récit et l'atteinte des objectifs fixés par le jeu, et d'autre part, le spectacle environnemental qui s'autonomise et devient un plaisir en soi. C'est aussi ce que soulignent King et Krzywinska lorsqu'ils expliquent que "de nombreux joueurs d'EverQuest et de jeux similaires passent beaucoup de temps à voyager pour voir les différents espaces du monde jouable, se comportant comme des touristes au sein de paysages virtuels ${ }^{22}$ ", ajoutant que «l'exploration, en soi, peut être une source substantielle de l'attrait de ce type de jeux $^{23}$ ». De son côté, Gordon Calleja ouvre In-Game, son ouvrage visant à clarifier les concepts d'immersion, de présence et d'incorporation, en racontant comment, lors de sa jeunesse à Malte, « les forêts et les chaînes de montagnes [constituaient] la matière même de la fantasy, et avoir la chance d'habiter ces paysages [...] formait un aspect séduisant des jeux numériques ${ }^{24}$ ». Le RPG japonais devenu culte, Earthbound (1995), fait d'ailleurs un clin d'œil amusant à ce type de plaisir en s'accompagnant d'une parodie de guide touristique décrivant ses différents lieux (banlieue coquette, désert, métropole, station balnéaire, forêt nordique...) et en intégrant au jeu lui-même un photographe qui tombe tout à coup du ciel pour immortaliser le passage des héros sur les sites les plus pittoresques. 
Il semble donc qu'il y ait, au-delà du plaisir compétitif de vaincre le jeu, au-delà du désir vectorisé, téléologique de finir le jeu, un plaisir de l'exploration pure, un plaisir esthétique et sensoriel de l'habitation et de l'errance au sein d'environnements virtuels. Ne retrouve-t-on pas, en littérature, ce type de plaisir, qui ne serait pas un plaisir de l'avancée et du progrès, de la résolution d'énigme, mais un plaisir de l'habitation d'un monde imaginaire, un plaisir immersif et sensuel autour duquel se structurerait un genre « environnemental » comme la fantasy?

\section{Habiter le texte, habiter le jeu}

Prix Nobel de littérature 2013, l'écrivaine Alice Munro décrit ainsi sa manière de lire, dans un essai où elle parle de sa vision de la nouvelle :

So obvisouly I don't take up a story and follow it as if it were a road, taking me somewhere, with views and neat diversions along the way. I go into it, and move back and forth and settle here and there, and stay in it for a while. It's more like a house ${ }^{25}$.

Bien que cette description ne puisse s'appliquer à toute lecture (elle semble particulièrement pertinente dans le cas de la relecture, crucial pour une conception de l'habitation textuelle), elle révèle la désirabilité de ce type de rapport esthétique. Munro décrit bien ce plaisir de la lecture qui échappe à la directionnalité du récit, au développement, au but à atteindre au bout de la route, pour se consacrer à une forme d'habitation. C'est en quelque sorte les chaînes de la succession, du syntagme que Munro rejette ici en faveur de l'élément paradigmatique, libéré des contraintes de la ligne temporelle.

Le projet New Eden - A book Celebrating Great Game Environment Design, développé en 2014 par trois étudiants de l'Université de Berlin (Christian Ernst, Alexander Venus et Robin Soyk), répond à une logique similaire; exposant des images tirées d'une variété d'environnements vidéoludiques et d'espaces virtuels, le livre d'art extrait ceux-ci de leur contexte original, où le joueur est trop souvent absorbé par les « missions » que lui donne le jeu pour entrer en état de contemplation esthétique. Cette même logique gouverne le travail du commissaire Jean-Jacques Launier pour son exposition «L'Art dans le jeu vidéo » (Art ludique-Le Musée, Paris, 25 septembre 2015 au 6 mars 2016), ainsi que plusieurs propositions en game art. Cette branche de l'art contemporain nous a en effet donné ces dernières décennies des exemples fascinants de l'attrait que peuvent avoir les environnements vidéoludiques libérés des contraintes du jeu et des actions qu'il nécessite. Un des premiers projets du pionnier du game art Miltos Manetas, SuperMario Sleeping (1997), est une vidéo d'art de type machinima, c'est-à-dire un film réalisé entièrement en utilisant un jeu vidéo. Exposé pour la première fois en 1997 au Institute of Contemporary Art de Londres, et pensé comme un clin d'œil au film Sleep d'Andy Warhol (1964), on peut voir, dans ce ready-made vidéoludique, le célèbre Mario (dans le jeu Super Mario 64, 1996) immobile au milieu d'un environnement verdoyant, trois papillons voletant doucement autour de lui, qui s'étire et baille, puis s'assoit dans l'herbe et dodeline de la tête, avant de s'étendre sur le côté pour dormir paisiblement. Cette routine est programmée dans le jeu original, et se déclenche lorsque le joueur laisse Mario immobile, mais elle est ici sortie de son contexte, nous permettant de nous concentrer sur l'aspect bucolique, paisible du jeu, sur le désir du joueur d'investir cet environnement édénique pour s'y reposer. Étienne Armand Amato explique bien 
comment la différence de pression entre un monde quotidien stressant et les mondes vidéoludiques enchanteurs et rassurants crée une "poussée immersive ${ }^{26}$ ». Il y a bien ici une habitation de l'environnement virtuel, qui devient havre de paix, refuge, protection, habitat, par la suspension de l'action et des objectifs à atteindre, ce qui permet une intensification des ressources attentionnelles allouées à l'environnement, à ses textures, couleurs et sons. Miltos Manetas prend ici le jeu vidéo à contre-emploi... mais est-ce vraiment le cas, ou exploite-t-il au contraire un potentiel inhérent au médium?

Entre rétrogaming et hackart, l'œuvre de Cory Arcangel, Super Mario Clouds (2002), modifie le code d'une cartouche originale de Super Mario Bros. (1985) afin d'enlever tous les éléments du jeu, sauf les nuages blanc flottants dans le ciel bleu du premier niveau. Généralement projeté sur plusieurs murs d'une galerie, ce travail soustractif permet de plonger le spectateur dans l'univers créé à l'origine par Shigeru Miyamoto et Takashi Tezuka, mais sur un mode contemplatif, bucolique, alors que cet univers est réduit à un élément simple (son ciel), qui concentre et met en évidence l'attrait immersif (ici d'ordre météorologique) de cet univers secondaire. Ce rapport au jeu est diamétralement opposé à celui qu'on trouve dans la pratique du speed run, où le joueur tente de traverser le jeu le plus rapidement possible. Dans ce dernier cas, le sens de ces nuages blancs, leur poids sensuel est atténué, voire neutralisé.

C'est ainsi que le très populaire RPG Skyrim, paru en 2011, s'il donne lieu à un type de jeu plutôt guerrier, en général, a aussi séduit largement pour ses longues séquences d'exploration, de promenades dans des environnements paisibles, se transformant au fil des saisons et de la succession des jours et des nuits. On trouve de nombreuses vidéos sur le $\mathrm{Web}^{27}$ qui témoignent de ce type d'interaction, entre exploration et habitation, plaisir immersif, purement sensoriel et esthétique. Les joueurs se plaisent ainsi à entrer dans un rapport esthétisant, géographique et climatique avec un monde dont, comme chez Tolkien, la densité historique est indéniable ${ }^{28}$.

\section{Narration et description}

En dépit du fait que les RPG puissent être catégorisés comme « jeux de progression », ce qui signifie, dans la typologie proposée par Jesper Juul, qu'ils sont structurés comme des séquences plus ou moins linéaires à travers lesquelles le joueur doit progresser (contrairement aux jeux d'émergence, comme les jeux de cartes, qui, à partir de quelques règles, peuvent générer un nombre presque infini de parties ${ }^{29}$ ), ces jeux fournissent aussi un véritable plaisir au flâneur musardant à travers leurs environnements. D'une manière similaire et comme nous l'avons vu avec Tolkien, la littérature fantasy, bien qu'elle s'apparente le plus souvent au genre épique de la quête, s'organise également autour de l'habitation de mondes secondaires. C'est ainsi que, tout en accommodant une esthétique temporelle, le texte et le jeu fantasy mettent l'accent sur la dimension spatiale, favorisant autant l'immersion sensorielle dans des environnements spectaculaires que l'absorption cognitive et émotionnelle dans les chaînes causales de l'intrigue. Cette tension peut être conceptualisée via l'opposition narratologique classique entre description et narration.

Dans les jeux vidéo de rôle, jouer pour gagner fait avancer le récit, alors qu'explorer les différents environnements, pour le simple plaisir de la découverte et de la contemplation esthétique, suspend sa progression. En ce sens, le jeu «compétitif » est 
lié à la résolution, c'est-à-dire à une téléologie, à une syntaxe narrative, alors que l'exploration a des affinités avec la description, avec la promenade à travers des paradigmes figuraux. L'implication du lecteur ou du joueur balance entre ces pôles, avec les exigences de progression définissant l'usage standard, le script comportemental dominant de la lecture et du jeu : se rendre à la fin de l'histoire, finir le livre ou le jeu. Ce script comportemental est subverti par la liberté et l'oisiveté de joueurs et de lecteurs qui "perdent leur temps» dans l'habitation des espaces fictionnels (notamment par la relecture ou le replay) et dans l'immersion touristique au sein du spectacle environnemental. C'est ainsi que la description devient dé-scription : destruction temporaire des forces téléologiques de la narration, échappée hors du temps ordonné dans celui d'un environnement dé-scripté, débarrassé des scripts qui règlent normalement notre rapport avec lui. L'élaboration informatique de la fantasy, par le jeu vidéo, intensifie l'attrait de ce mode d'implication.

Bien entendu, les deux modes d'implication sont complémentaires, et la lecture passe sans cesse de la progression narrative à l'habitation descriptive. Pour mieux comprendre cette dernière, il faut souligner avec Philippe Hamon le rôle central, au sein de la description, du nom, du substantif, que l'on peut considérer comme la racine dénominative qui, en s'associant une série de prédicats, s'étoffe, s'étire, croit, fleurit ${ }^{30}$. Le nom porte en lui une autonomie, que ne possèdent pas l'adjectif, l'adverbe ou même le verbe (soumis au sujet et à son objet). Car si le nom, qui désigne ou évoque, s'intègre le plus souvent dans une phrase qui le définit, il menace toujours de quitter cette phrase pour construire son propre temple. Il y a des cultes du nom: Gertrude Stein ("A rose is a rose is a rose ", Geography and Plays, 1922), Francis Ponge (Le Parti pris des choses, 1942). Le nom est souverain, il porte l'ombre d'une présence du monde, la saveur de l'objet absent. La description reproduit à plus grande échelle cette logique du nom souverain, procurant - comme les projets en game art évoqués plus haut - un plaisir « inutile » et spectaculaire, souvent environnemental, immersif, sensuel et détaché des chaînes de l'action.

\section{Du nom souverain au spectacle environnemental}

Chez Tolkien, le nom est créateur d'un monde secondaire enchanteur. Il contient la chose matérielle, qu'il fait exister glorieusement. "Tree and bird, water and stone", l'écriture tolkienienne se délecte de l'objet environnemental, valable en soi, indépendamment d'une logique narrative. Comme le remarque Tom Shippey, cela est particulièrement vrai des noms propres, qui sont en relation unique avec ce qu'ils désignent ${ }^{31}$. Rares dans The Hobbit, les noms de lieux pullulent dans The Lord of the Rings et dans le Silmarillion, créant un fort sentiment-de-monde : puissance de l'onomastique tolkienienne. Cette puissance repose notamment sur l'intérêt professionnel de Tolkien pour l'étymologie et l'histoire des noms de sa région (Oxfordshire), dont il tirera nombre des paysages qui dominent The Lord of the Rings. Ce serait d'ailleurs une visite des gorges de Cheddar, dans le sud du Somerset, qui aurait inspiré la description des grottes d'Aglarond, que nous évoquerons maintenant en guise de conclusion ${ }^{32}$. Notons au passage l'importance des pratiques de plein air dans l'essor de la fantasy, des randonnées du professeur d'Oxford aux sorties spéléologiques de l'auteur de Colossal Cave Adventure, évoquées précédemment, en passant par les treks asiatiques des 
pionniers du "Livre dont vous êtes le héros", Ian Livingstone et Steve Jackson, qui auront inspiré à chacun ce qu'ils considèrent comme leur meilleur ouvrage ${ }^{33}$.

The Lord of the Rings compte peu de descriptions aussi lyriques et aussi énergiques que le portrait que fait Gimli des somptueuses grottes d'Aglarond, lors du chapitre "The Road to Isengard ». On touche ici à l'hypotypose : la description rend présent son objet non seulement à nos yeux, mais à ceux de l'elfe Legolas, à qui s'adresse la tirade du nain et qui devient ici lecteur modèle. Intégrant le lieu dans une géographie culturelle (humaine et naine) qui renforce le sentiment de n'apercevoir que des bribes d'un monde plus vaste dont la vie déborde le récit en cours, la description que fait Gimli envisage le dépassement de la perspective militaire par un tourisme du recueillement et de la contemplation (une fois la guerre de l'anneau terminée, les nains viendront en pèlerinage contempler cette merveille du monde naturel). La beauté de la caverne est alors pensée comme souveraine, autonome de toute considération utilitaire ou guerrière, élevée en spectacle environnemental, en objet esthétique. Il y a ici tension productive : si, d'une part, l'image merveilleuse joue un rôle dans le développement du récit (guerrier), lui donnant comme but la mise en place d'un monde apaisé et touristique, d'autre part, l'immensité de cette image tend à la détacher de l'ensemble.

L'énergie, l'energeia qui crée ici l'hypotypose est celle, esthétique et affective, du bouleversement par la beauté et la merveille naturelle. En effet, Gimli commence sa tirade sur le mode affectif, confiant à Legolas que son cœur est encore plein de la beauté des grottes d'Aglarond (« my heart is still full of it »). Il enchaîne ensuite :

And, Legolas, when the torches are kindled and men walk on the sandy floors under the echoing domes, ah! then, Legolas, gems and crystals and veins of precious ore glint in the polished walls; and the light glows through folded marbles, shell-like, translucent as the living hands of Queen Galadriel. There are columns of white and saffron and dawn-rose, Legolas, fluted and twisted into dreamlike forms; they spring up from many-colored floors to meet the glistening pendants of the roof: wings, ropes, curtains fine as frozen clouds; spears, banners, pinnacles of suspended palaces! Still lakes mirror them : a glimmering world looks up from dark pools covered with clear glass ; cities, such as the mind of Durin could scarce have imagined in its sleep, stretch on through avenues and pillared courts, on into the dark recesses where no light can come. And plink! A silver drop falls, and the round wrinkles in the glass make all the towers bend and waver like weeds and corals in a grotto of the sea ${ }^{34}$.

31 La structure rythmique de ce passage très musical est complexe, reposant notamment sur les interjections "Legolas", attirent l'oreille du lecteur, appelé à écouter attentivement lui aussi. On peut penser que cette modulation de l'attention favorise l'immersion. Le «ah!», marqueur d'affectivité, syncope le flot de la phrase, qui s'étire ensuite en énumérations de noms (gems and crystals and veins of precious ore). Le minéral devient souple et translucide, vivant, multicolore, il s'élève, se tord en une phrase qui accumule deux points-virgules, un deux points, un point d'exclamation. Les énumérations créent un monde fantastique de formes aériennes (wings, ropes, curtains, frozen clouds), qui se complique et se redouble dans l'aquatique et l'urbain (still lakes, dark pools, cities, avenues and pillared courts) pour un spectacle visuel doté d'agentivité (a world looks up), soudainement interrompu par l'onomatopée «And plink!». Le son mimé nous emporte dans le présent de la contemplation, nous immergeant finalement dans une grotte océanique (like weeds and corals in a grotto of the sea).

L'analyse de ce passage pourrait se prolonger, mais l'essentiel est de comprendre pourquoi une telle intensité stylistique et rhétorique est ici concentrée. Cette pièce 
brillante, ce bijou textuel, vibrant de noms évocateurs, ornementaux, est un cadeau que Gimli fait à Legolas, et un cadeau que Tolkien fait à son lecteur, après un épisode particulièrement éprouvant. Ce cadeau fait partie d'une économie esthétique où le spectacle environnemental tend à se détacher du récit, comme un surplus, un ornement excessif. Ce caractère excessif semble fonder en partie les critiques adressées à l'usage comparable que fait le jeu vidéo de scènes cinématiques spectaculaires, souvent données en récompense au joueur après une épreuve difficile ${ }^{35}$.

Le développement de l'informatique et du jeu vidéo aura donc sélectionné, au sein de la tradition littéraire fantasy, un certain type de rapport esthétique - l'exploration et l'habitation d'environnements spectaculaires - qu'il intensifie et valorise rétrospectivement. Permettant aux joueurs de vagabonder au sein de mondes secondaires qu'imaginait déjà la littérature, la technique vidéoludique élargit notre habitat quotidien, nous propose des refuges apaisants, détachés des exigences de l'action, favorisant ainsi des formes de contemplation environnementales. L'avenir nous dira si ce type d'engagement, privilégié par le jeu vidéo de rôle, deviendra en retour plus commun dans les récits littéraires (avec des formes d'habitation qu'on retrouve par exemple dans un roman comme House of Leaves de Mark Z. Danielewski, 2000) et dans le monde de l'art (où les environnements immersifs sont en effet très en vogue depuis le début du Xxi siècle), ou bien si, au contraire, la littérature délaissera l'immersion environnementale pour se spécialiser dans d'autres dimensions de l'expérience esthétique.

\section{BIBLIOGRAPHIE}

"A Walk Through Skyrim : The Rift », YouTube. [En ligne] https://www.youtube.com/watch? $\mathrm{v}=\mathrm{ONYCv35t04s}$ [consulté le 21 novembre 2018].

AARSETH Espen, «Allegories of Space. The Question of Spatiality in Computer Games ", in Friederich VON BORRIES, Steffen P. WALZ et Matthias BöTTGER (dir.), Space Time Play, Bâle, Birkhäuser, 2007, p. 44-47.

Aмато Étienne Armand, «L'immersion par le jeu vidéo : origine et pertinence d'une métaphore signifiante », in Bernard Guelton (dir.), Les Figures de l'immersion, Rennes, Presses universitaires de Rennes, 2013, p. 39-59.

CALLEJA Gordon, In Game. From Immersion to Incorporation, Cambridge, MIT Press, 2011.

CARPENTER Humphrey, The Letters of J.R.R. Tolkien, Boston, Houghton Mifflin Harcourt, 2014.

ColuINS Christopher, Paleopoetics. The Evolution of the Preliterate Imagination, New York, Columbia University Press, 2013.

GREEN Jonathan, You are the Hero. A History of Fighting Fantasy Gamebooks, Bucks, Snowbooks, 2014.

HAMON Philippe, Introduction à l'analyse du descriptif, Paris, Hachette, 1981. 
HAYLES N. Katherine, Electronic Literature. New Horizons for the Literary, Notre Dame, University of Notre Dame Press, 2008.

HOWARD Robert Ervin, « The Hyborian Age », The Complete Chronicles of Conan [1936], Londres, Gollancz, 2006, p. 1-20.

Horowitz Michael et PALmer Cynthia (dir.), Moskha. Aldous Huxley's Classic Writings on Psychedelics and the Visionary Experience [1977], Rochester, Park Street Press, 1999.

JACKSON Steve et LIVINGSTONE Ian, The Warlock of Firetop Mountain [1982], 25 ${ }^{\text {th }}$ anniversary edition, Cambridge, Wizard Books, 2007.

JENKINS Henry, « Game Design as Narrative Architecture », in Noah WARDRIP-FRUIN et Pat HARRIGAN (dir.), First person. New media as story, performance, and game, Cambridge, MIT Press, 2004, p. $118-130$.

JuUL Jesper, Half-Real. Videogames Between Real Rules and Fictional Worlds, Cambridge, MIT Press, 2005.

KING Brad et BoRland John, Dungeons and Dreamers. The Rise of Computer Game Culture from Geek to Chic, Emeryville, McGraw-Hill/Osborne, 2003.

KING Geoff et KRZYwinSKA Tanya, Tomb Raiders and Space Invaders. Videogames Froms and Contexts, Londres/New York, I.B. Taurus, 2006.

KLEVIER Rune, «In Defense of Cutscenes », in Frans MAYRA (dir.), Proceedings of Computer Games and Digital Cultures Conference, Tampere, Tampere University Press, 2002.

LEROI-GOURHAN André, Le Geste et la Parole, vol. 1, Technique et langage, Paris, Albin Michel, 1964.

MACÉ Marielle, Façons de lire, manières d'être, Paris, Gallimard, 2011.

MoNTANI Pietro, Bioesthétique. Sens commun, technique et art à l'âge de la globalisation, Paris, Vrin, 2013.

MonTFORT Nick, Twisty Little Passages. An Approach to Interactive Fiction, Cambridge, MIT Press, 2005.

MUnRo Alice, « What is real ? ", in Tammy ROBERTS, Mical MOSER, Don LEPAN, Julian GAUNCE et Laura BUZZARD (dir.), The Broadview Anthology of Expository Prose. Second Edition [1982], Calgary, Broadview Press, 2011.

NeWMAN James, Playing With Videogames, Londres/New York, Routledge, 2008.

RYAN Marie-Laure, Avatars of Story, Minneapolis/Londres, University of Minnesota Press, 2006.

SHIPPEY Tom, J.R.R. Tolkien. Author of the Century, Londres, Harper Collins, 2000.

THÉRRIEN Carl, Illusion, idéalisation, gratification. L'immersion dans les univers de fiction à l'ère du jeu vidéo, thèse présentée comme exigence partielle du doctorat en sémiologie, Montréal, Université du Québec à Montréal, 2011.

TOLKIEN John Ronald Reuel, Tree and Leaf, Boston, Houghton Mifflin Company, 1965.

ToLKIEN John Ronald Reuel, The Two Towers [1954], Boston, Houghton Mifflin Company, 1982.

TUAN Yu-Fi, Escapism, Baltimore, Johns Hopkins University Press, 1998.

VERCRUYSSE Thomas, « Façons de lire, manières de devenir. La lecture comme occasion d'une éthopoétique », Épistémocritique, 2015. [En ligne] http://epistemocritique.org/facons-de-lire- 
manieres-de-devenir-la-lecture-comme-occasion-dune-ethopoetique/ [consulté le 14 décembre 2015].

\section{NOTES}

1. N. Katherine Hayles, Electronic Literature. New Horizons for the Literary, Notre Dame, University of Notre Dame Press, 2008, p. 118. N. B. : Les citations de cet article proviennent essentiellement d'ouvrages en anglais et ont été traduites par l'auteur. Les citations longues d'écrivains (Tolkien et Munro) sont données en version originale.

2. Dans Dungeons and Dreamers, King et Borland évoquent cette histoire particulière à travers la biographie de Richard Garriot (le créateur de la série de jeux vidéo Ultima), décrivant notamment son passage dans un camp de programmation alors qu'il est encore adolescent, en 1977 (Brad King et John Borland, Dungeons and Dreamers. The Rise of Computer Game Culture from Geek to Chic, Emeryville, McGraw-Hill/Osborne, 2003).

3. Marie-Laure Ryan, Avatars of Story, Minneapolis/Londres, University of Minnesota Press, 2006, p. 127.

4. James Newman, Playing With Videogames, Londres/New York, Routledge, 2008, p. 48 sq.

5. Voir à ce sujet l'essai d'Aldous Huxley datant de 1977. Michael Horowitz et Cynthia Palmer (dir.), Moskha. Aldous Huxley's Classic Writings on Psychedelics and the Visionary Experience, Rochester, Park Street Press, 1999, p. 119, ou les travaux du géographe Yu-Fi Tuan, Escapism, Baltimore, Johns Hopkins University Press, 1998.

6. Brad King et John Borland, Dungeons and Dreamers. The Rise of Computer Game Culture from Geek to Chic, Emeryville, McGraw-Hill/Osborne, 2003, p. 21.

7. Pietro Montani, Bioesthétique. Sens commun, technique et art à l'âge de la globalisation, Paris, Vrin, 2013, p. 106.

8. André Leroi-Gourhan, Le Geste et la Parole, vol. 1, Technique et langage, Paris, Albin Michel, 1964.

9. Christopher Collins, Paleopoetics. The Evolution of the Preliterate Imagination, New York, Columbia University Press, 2013, p. 133.

10. Christopher Collins, Paleopoetics. The Evolution of the Preliterate Imagination, New York, Columbia University Press, 2013, p. 154.

11. Au sujet de cette oscillation, voir les travaux de Marielle Macé, Façons de lire, manières d'être, Paris, Gallimard, 2011, p. 53, et de Thomas Vercruysse, « Façons de lire, manières de devenir. La lecture comme occasion d'une éthopoétique», Épistémocritique, 2015. [En ligne] http:// epistemocritique.org/facons-de-lire-manieres-de-devenir-la-lecture-comme-occasion-duneethopoetique/ [consulté le 14 décembre 2015].

12. Yu-Fi Tuan, Escapism, Baltimore, Johns Hopkins University Press, 1998, p. 38.

13. Robert Ervin Howard, «The Hyborian Age », The Complete Chronicles of Conan [1936], Londres, Gollancz, 2006, p. 1-20.

14. John Ronald Reuel Tolkien, Tree and Leaf, Boston, Houghton Mifflin Company, 1965, p. 51.

15. John Ronald Reuel Tolkien, Tree and Leaf, Boston, Houghton Mifflin Company, 1965, p. 9.

16. Espen Aarseth, "Allegories of Space. The Question of Spatiality in Computer Games ", in Friederich von Borries, Steffen P. Walz et Matthias Böttger (dir.), Space Time Play, Basel, Birkhäuser, 2007, p. 44.

17. Henry Jenkins, "Game Design as Narrative Architecture », in Noah Wardrip-Fruin et Pat Harrigan (dir.), First person. New media as story, performance, and game, Cambridge, MIT Press, 2004, p. 121.

18. Je remercie l'évaluateur anonyme qui m'a rappelé l'importance de ces « jeux non spatiaux ».

19. Geoff King et Tanya Krzywinska, Tomb Raiders and Space Invaders. Videogames Froms and Contexts, Londres/New York, I.B. Taurus, 2006, p. 156. 
20. Voir Brad King et John Borland, Dungeons and Dreamers. The Rise of Computer Game Culture from Geek to Chic, Emeryville, McGraw-Hill/Osborne, 2003, p. 31.

21. Nick Montfort, Twisty Little Passages. An Approach to Interactive Fiction, Cambridge, MIT Press, 2005, p. 4.

22. Geoff King et Tanya Krzywinska, Tomb Raiders and Space Invaders. Videogames Froms and Contexts, Londres/New York, I.B. Taurus, 2006, p. 156.

23. Geoff King et Tanya Krzywinska, Tomb Raiders and Space Invaders. Videogames Froms and Contexts, Londres/New York, I.B. Taurus, 2006, p. 91.

24. Gordon Calleja, In-Game. From Immersion to Incorporation, Cambridge, MIT Press, 2011, p. 1.

25. Alice Munro, «What is real?» [1982], in Tammy Roberts, Mical Moser, Don LePan, Julian Gaunce et Laura Buzzard (dir.), The Broadview Anthology of Expository Prose. Second Edition, Calgary, Broadview Press, 2011, p. 267.

26. Étienne Armand Amato, "L'immersion par le jeu vidéo: origine et pertinence d'une métaphore significative », in Bernard Guelton (dir.), Les Figures de l'immersion, Rennes, Presses Universitaires de Rennes, 2014, p. 54.

27. Par exemple "A Walk Through Skyrim: The Rift», YouTube. [En ligne] https:// www.youtube.com/watch?v=ONYCv35t04s [consulté le 21 novembre 2018].

28. Pour une étude des stratégies de construction de monde dans la série The Elder Scrolls, dont Skyrim est le cinquième opus, voir Carl Thérrien, Illusion, idéalisation, gratification. L'immersion dans les univers de fiction à l'ère du jeu vidéo, thèse présentée comme exigence partielle du doctorat en sémiologie, Montréal, UQAM, 2011.

29. Jesper Juul, Half-Real. Videogames Between Real Rules and Fictional Worlds, Cambridge, MIT Press, 2005.

30. Philippe Hamon, Introduction à l'analyse du descriptif, Paris, Hachette, 1981, p. 185-186.

31. Tom Shippey, J.R.R. Tolkien. Author of the Century, Londres, Harper Collins, 2000, p. 57.

32. Humphrey Carpenter, The Letters of J.R.R. Tolkien, Boston, Houghton Mifflin Harcourt, 2014, p. 407.

33. Steve Jackson et Ian Livingstone, The Warlock of Firetop Mountain [1982], 25 $5^{\text {th }}$ anniversary edition, Cambridge, Wizard Books, 2007, p. 220.

34. John Ronald Reuel Tolkien, The Two Towers [1954], Boston, Houghton Mifflin Company, 1982, p. 152-153.

35. Pour une présentation du débat à propos des scènes cinématiques, voir Rune Klevier, "In Defense of Cutscenes ", in Frans Mayra (dir.), Proceedings of Computer Games and Digital Culture Conference, Tampere, Tampere University Press, 2012.

\section{RÉSUMÉS}

Dès les années 1970, le jeu vidéo s'intéresse de manière déterminante au récit. Quels impacts cette forme d'art, native d'un milieu technique marqué par l'informatique, a-t-elle sur notre manière d'appréhender le texte littéraire, de faire l'expérience du récit et de la fiction au $\mathrm{XXI}^{\mathrm{e}}$ siècle ? Nous interrogeons ces impacts en explorant le genre de la fantasy, que partagent le jeu vidéo de rôle et la forme romanesque, et qui définit une matrice d'influence s'organisant autour d'un principe esthétique, celui de l'exploration, et de l'habitation d'univers secondaires que rend possible l'immersion environnementale. Nous verrons notamment que la fantasy tend à 
libérer la description des exigences de la narration et à ménager des espaces de souveraineté au spectacle environnemental.

\section{INDEX}

Mots-clés : environnement, fantasy, immersion, jeu vidéo, lecture, Tolkien

\section{AUTEUR \\ PIERRE-LOUIS PATOINE}

Pierre-Louis Patoine est maître de conférences à la Sorbonne Nouvelle, où il codirige avec Liliane Campos le groupe de recherche [Science/Littérature] (litorg.hypotheses.org). Corédacteur en chef de la revue Épistémocritique, ses travaux se situent à l'intersection des sciences du vivant et des études littéraires. Il a publié divers articles portant sur la littérature américaine contemporaine, la biosémiotique et la neuro-esthétique. Son premier ouvrage, intitulé Corps/ texte. Pour une théorie de la lecture empathique est paru en 2015 chez ENS Éditions. 